\title{
Synthesis and Characterization of pH-Sensitive Thiol-Containing Chitosan Beads for Controlled Drug Delivery Applications
}

\author{
R. Jayakumar, R. L. Reis, and J. F. Mano \\ 3Bs Research Group-Biomaterials, Biodegradables and Biomimetics, University of Minho, \\ 4710-057 Braga, Portugal; and Department of Polymer Engineering, University of Minho, \\ Campus of Azurém, 4800-058 Guimarães, Portugal
}

The aim of this study was to develop chitosan-based materials in drug delivery systems possessing covalent attachment of thiol moieties. Thiol-containing chitosan (TCS), found to be soluble in water, was synthesized by graft copolymerization technique. The TCS beads were prepared by using tripolyphoshate, at pH 4.0. The morphology of TCS beads was examined by scanning electron microscopy. The in vitro drug release behavior was studied in phosphate buffer solution at various $\mathbf{p H}$, using indomethacin as a model drug at two different concentrations $(0.3$ and $0.6 \%$ w/w). The release amounts of indomethacin from TCS beads were higher increasing $\mathrm{pHs}$ in the dissolution medium. The release rate of indomethacin at $\mathrm{pH} 7.4$ was higher than the release rate at $\mathrm{pH} 1.4$ due to ionization of thiol groups and high solubility of indomethacin in an alkaline medium. These results indicated that the TCS beads may become a delivery system for the controlled release of different drugs wherever pH sensitive mechanics might be useful. This is especially applicable in cases when it is important to minimize drug release in acidic sites, such as in the stomach.

Keywords Beads, Indomethacin, Thiol-Containing Chitosan, TPP

Chitosan (CS) is obtained by alkaline deacetylation of chitin, which is one of the most abundant polysaccharides in nature. Shell wastes of shrimp, lobster, and crab are the main industrial sources of chitin (Muzzarelli 1997; Sorlier et al. 2001). CS is a polysaccharide consisting of copolymers of glucosamine and $\mathrm{N}$ acetylglucosamine. The primary amino group accounts for the possibility of relatively easy chemical modification of chitosan and salt formation with acids. At acidic $\mathrm{pH}$ the amino groups are

Received 5 September 2005; accepted 5 December 2005.

R. Jayakumar thanks the Portuguese Foundation for Science and Technology for providing him with a Post-Doctoral Research Fellowship (SFRH/BPD/14670/2003). This work was partially supported by the FCT Foundation for Science and Technology, through funds from the POCTI and/or FEDER programs, and by the European Unionfunded STREP Project HIPPOCRATES (NMP3-CT-2003-505758).

Address correspondence to J. F. Mano, 3Bs Research Group, University of Minho, Braga, Portugal. E-mail: jmano@dep.uminho.pt protonated, which promotes solubility, whereas chitosan is insoluble at alkaline and neutral pH (Fini and Orienti 2003). Because of its favorable properties, such as enzymatic biodegradability, nontoxicity, and biocompatibility (Muzzarelli 1997; Sorlier et al. 2001; Fini and Orienti 2003), chitosan has received considerable attention as a novel carrier in drug delivery systems and has been included in the European Pharmacopoeia since 2002. So far, chitosan has been utilized in various fields of pharmaceutical dosage forms, such as tablets, gels, and microspheres, and as mucoadhesive and/or permeation-enhancing excipient for oral, nasal, ocular, and buccal drug delivery (Takeuchi, Yamamoto, and Kawashima 2001; Felt et al. 1999; Senel et al. 2000), and in nonviral gene delivery (Borchard 2001; Liu and Yao 2002).

Interest is a growing in the chemical modification of CS to improve its solubility and widen its applications (Sugimoto, Morimoto, and Sashiwa 1998; Sashiwa and Shigemasa 1999; Heras, Rodriguez, and Ramos 2001). Among various methods, graft copolymerization is most attractive because it is a useful technique for modifying the chemical and physical properties of natural polymers. CS bears two types of reactive groups that can be grafted. First, the free amino groups on deacetylated units and second, the hydroxyl groups on the $\mathrm{C}_{3}$ and $\mathrm{C}_{6}$ carbons deacetylated units. Grafting of CS allows the formation of functional derivatives by covalent binding of a molecule, the graft, onto the CS backbone. Recently different researchers showed that after primary deviation followed by graft modification, CS would obtain much improved water solubility and bioactivities such as antibacterial and antioxidant properties (Xie et al. 2001, 2002; Jayakumar et al. 2005). Grafting CS is a common way to improve chitosan properties such as increasing chelating (Yang and Yuan 2001) or complexation properties (Chen and Wang 2001), bacteriostatic effect (Jung et al. 1999), or enhancing adsorption properties (Kotze et al. 1997; Thanou, Verhoef, and Junginger 2001). Although the grafting of CS modifies its properties, it is possible to maintain some interesting characteristics such as mucoadhesivity (Hoffman et al. 1997), biocompatibility (Tasker et al. 1998; Ono et al. 2000), and biodegradability (Singh and Ray 1998). 
When compared with other modified-chitosan materials, thiolated chitosans have numerous advantageous features, such as significantly improved mucoadhesive and permeation enhancing properties (Schnurch and Hornof 2001; Kast and Schnurch 2001; Hornof, Kast, and Schnurch 2003; Schnurch, Brandt, and Clausen 1999, 2003; Clausen, Kast, and Schnurch 2002). Moreover, solutions of thiolated chitosans display in situ gelling properties at physiological $\mathrm{pH}$ values (Hornof et al. 2003). The strong cohesive properties of thiolated chitosans make them highly suitable carriers for controlled drug release applications (Schnurch et al. 1999; Kast et al. 2002).

However, to our knowledge no work has been published on thiolated-chitosan particles or beads and its applications in the delivery of drugs and other substances. In this work, thiolactic acid was grafted into chitosan by using 1-ethyl-3-(3dimethylaminopropyl) carbodiimide by means of coupling reaction. The grafted chitosan was used for the preparation of beads using tripolyphosphate to improve using this controlled release system in contact with a gastric fluid, for instance. The morphological features of the system, swelling properties, and the in vitro drug release profiles of the prepared beads have been evaluated.

\section{MATERIALS AND METHODS}

Chitosan (deacetylation degree 75-85\%), tripolyphosphate (TPP), and thiolactic acid were received from Aldrich (Milwaokee, WI, USA). 1-ethyl-3-(3-dimethylaminopropyl) carbodiimide (EDC) was received from Alfa Aesar Company (Ward Hill, MA, USA). Indomethacin drug was purchased from Fluka (Buchs, Switzerland). All other materials used were analytical grade.

\section{Synthesis of Thiol-Containing Chitosan (TCS)}

CS $(0.1650 \mathrm{~g})$ was dissolved in $1 \% \mathrm{w} / \mathrm{w}$ acetic acid solution. Then thiolactic acid $(0.1055 \mathrm{~g})$ was dissolved in water and then added dropwise to the CS solution. Then, EDC (0.1552 g) was added to the above reaction mixture. The $\mathrm{pH}$ of the solution was maintained at 5.0. The reaction was conducted at $50^{\circ} \mathrm{C}$ for $7 \mathrm{hr}$. Then the reaction mixture was poured into acetone solution $(200 \mathrm{~mL})$ for precipitation. The precipitate was washed repeatedly with ethanol and acetone. The product was dried in vacuum at room temperature. The synthesis of TCS was shown in Diagram 1.

\section{Preparation of TCS Beads}

Thiol-containing chitosan TCS beads were prepared by using ionotropic gelation process with counter polyanion TPP. TCS $(0.7006 \mathrm{~g})$ was dissolved in $10 \mathrm{ml}$ of a $2 \% \mathrm{w} / \mathrm{w}$ acetic acid solution and stirred for 1 day to obtain a transparent and homogeneous solution. The TCS solution with or without drug was dropped through a syringe needle $(0.5 \mathrm{~mm}$ in diameter $)$ into the $10 \%$ TPP aqueous phase. Solidified white beads were formed immediately and allowed to stand for $1 \mathrm{hr}$ in the solution with
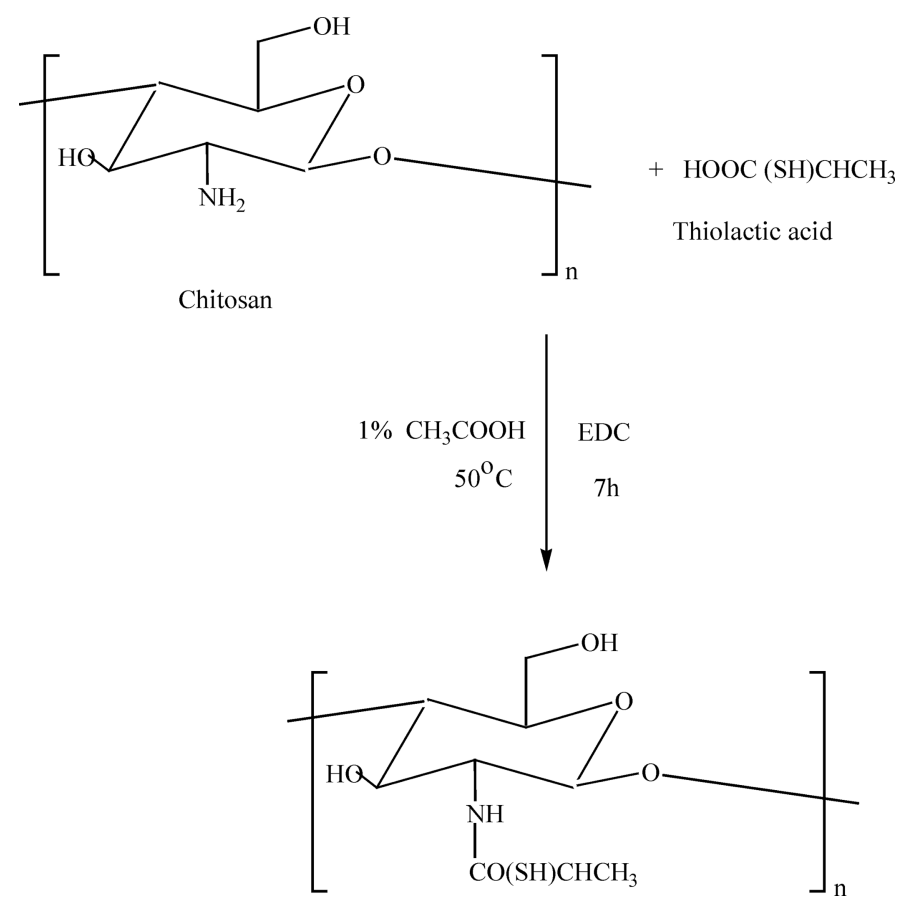

DIAG. 1. Synthesis of thiol-containing chitosan.

occasional agitation at room temperature. The $\mathrm{pH}$ of the TPP solution was adjusted to $\mathrm{pH} 4.0$ with a $1 \mathrm{M} \mathrm{HCl}$ solution. The gel beads were filtered, washed with distilled water repeatedly, and dried under vacuum at room temperature for 2 days. For the preparation of drug-loaded TCS beads two different amounts of indomethacin (IM) $(0.3$ and $0.6 \%$ w/w with respect to TCS solution) were dispersed in $10 \mathrm{~g}$ of TCS solution for 1 day. IM is a nonsteroid anti-inflammatory drug (NSAID) used to treat arthrthitis (Mi et al. 2002a). The advantages of such controlled release formulations containing NSAID over the conventional dosage forms were reported earlier (Kulkarni et al. 1999, 2001). Such formulations help to minimize the serious gastric irritation side effects of the conventional-dosage NSAID formulations. CS beads with or without IM were prepared from the same procedure.

\section{Swelling Tests}

Dried CS and TCS beads were carefully weighed and immersed in phosphate buffer media $(50 \mathrm{~mL})$ with $\mathrm{pH}$ values at 1.4 and 7.4 at $37^{\circ} \mathrm{C}$. At predetermined time intervals, swollen beads were taken out, and the excess water was blotted with filter paper from the surface, and then weighed on a sensitive balance. The following equation was used to determine the swelling degree.

$$
\text { Swelling degree }[\%]=\left\{\left(X_{w}-X_{d}\right) / X_{d}\right\} \times 100
$$

Here $X_{d}$ and $X_{w}$ represent the mass of dry and swollen beads, respectively. 


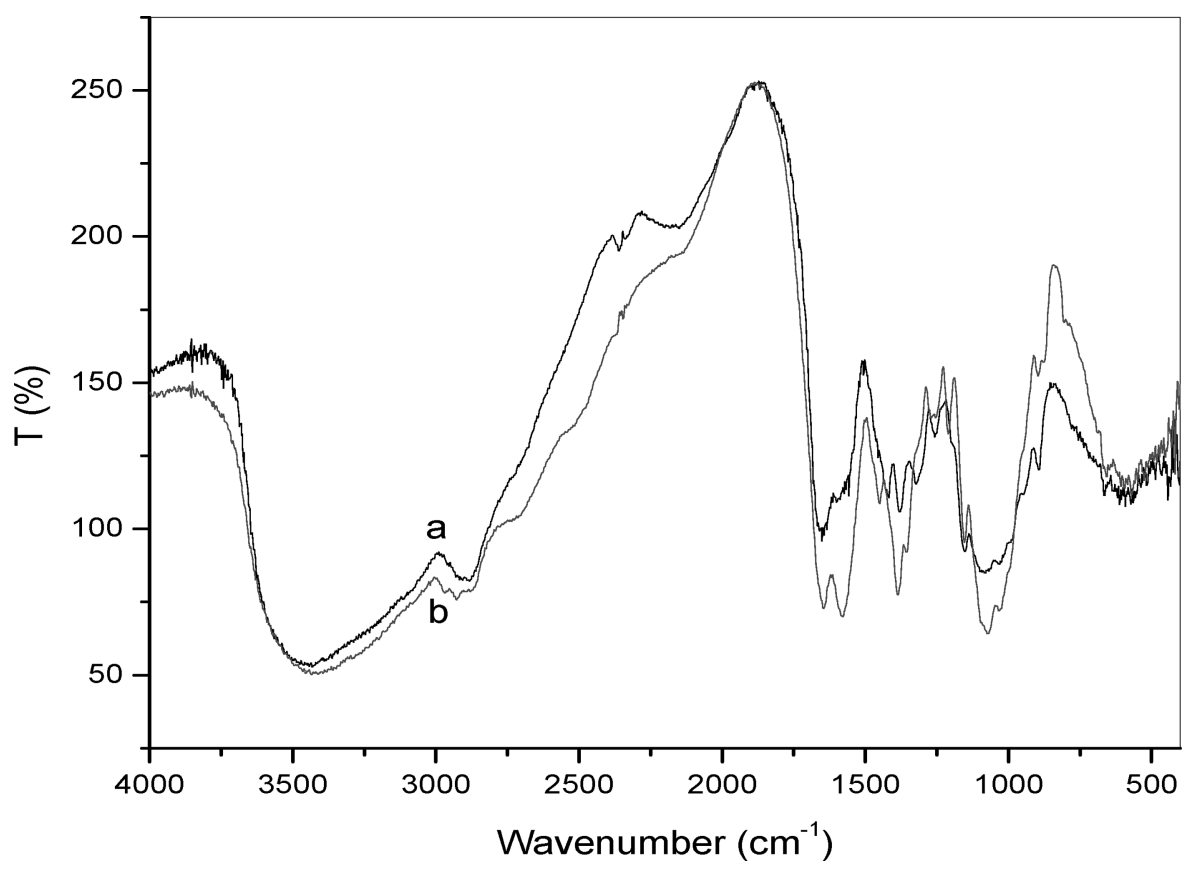

FIG. 1. FTIR spectrum of (a) CS (b) TCS.

\section{Determination of Drug-Loading Efficiency}

During the bead preparation process, the aqueous phase was collected, and the drug content in the aqueous phase was determined by ultraviolet spectrophotometer at $320 \mathrm{~nm}$. Then the loading efficiency during bead preparation process was calculated by the following formula:

$$
\text { Loading efficiency }[\%]=\{(m-c \times v) / m\} \times 100
$$

Where $m, c$, and $v$ represent the initial drug (IM) mass in beads (TCS) droplet, the IM concentrations in TPP solution, and the volume of TPP solution, respectively.

\section{In Vitro Drug Release Studies}

The in vitro release tests were carried out on all formulations of drug-loaded CS beads, and TCS beads were suspended in $100 \mathrm{~mL}$ of phosphate buffer at various $\mathrm{pH}$ at $37^{\circ} \mathrm{C}$ and placed in an incubated shaker at $120 \mathrm{rpm}$. At predetermined time intervals, $1 \mathrm{~mL}$ aliquot of sample was withdrawn and after suitable dilution, the concentration of drug released was monitored by ultraviolet spectrophotometer at $320 \mathrm{~nm}$. The dissolution medium was replaced with fresh buffer to maintain the total volume. The drug release percent can be determined by the following equation:

$$
\text { Drug release }[\%]=R_{t} / L \times 100
$$

Where $L$ and $R_{t}$ represent the initial amount of drug loaded and the cumulative amount of drug released at time $t$, respectively.

\section{Instruments}

The surface morphology of samples was analyzed by scanning electron microscopy (Leica Cambridge S360 microscope, UK). The drug release was monitored by ultraviolet spectrophotometer (Shimadzu UV-1601, Japan). The infrared spectrum was recorded with a double-beam Perkin-Elmer 1600 FTIR spectrophotometer.

\section{RESULTS AND DISCUSSION}

\section{Synthesis of TCS}

Thiolactic acid was attached covalently to the primary amino group of chitosan under the formation of amide bonds as shown in Diagram 1. The carboxylic acid moieties of thiolactic acid were activated by EDC forming as O-acylurea derivative as intermediate product that reacts with the primary amino groups of chitosan. The TCS was found to be soluble in both water and acetic acid. The yield of the TCS was $86 \%$. The FTIR spectrum of TCS and CS is shown in Figure 1. The FTIR spectrum of CS (Figure 1a) showed a broad -OH stretching absorption band between 3450 and $3100 \mathrm{~cm}^{-1}$ and the aliphatic C-H stretching between 2990 and $2850 \mathrm{~cm}^{-1}$. As the $-\mathrm{OH}$ stretching band and the aliphatic $\mathrm{C}-\mathrm{H}$ stretching band were appeared between 3450 and $2850 \mathrm{~cm}^{-1}$ in the spectrum, Another major absorption band between 1220 and $1020 \mathrm{~cm}^{-1}$ represented the free primary amino group $\left(-\mathrm{NH}_{2}\right)$ at $\mathrm{C}_{2}$ position. The peak at $1647 \mathrm{~cm}^{-1}$ represented acetylated amino group of chitin, which indicated that the sample is not fully deacetylated. The peak at $1384 \mathrm{~cm}^{-1}$ represents the $-\mathrm{C}-\mathrm{O}$ stretching of primary alcoholic group 


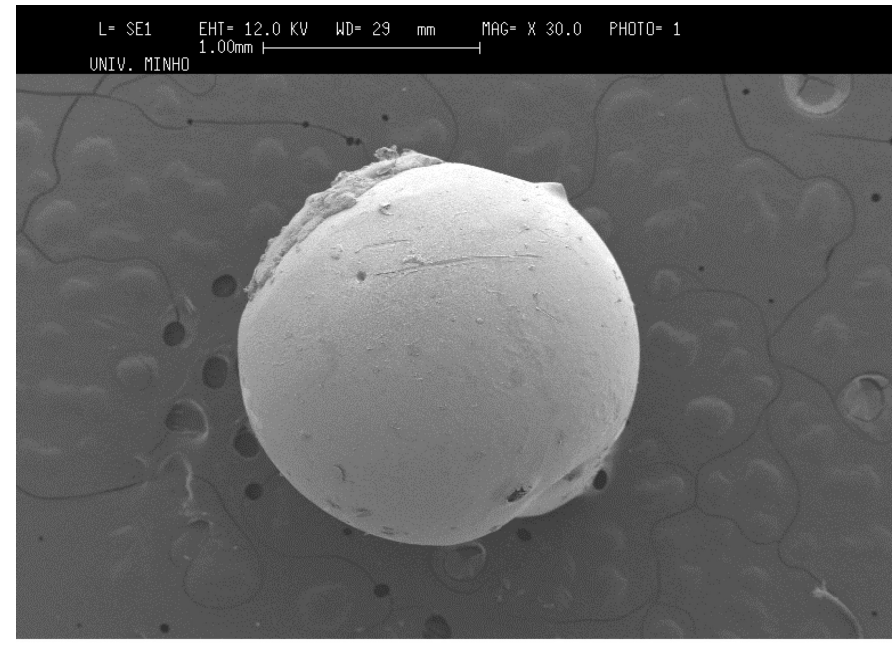

(a)

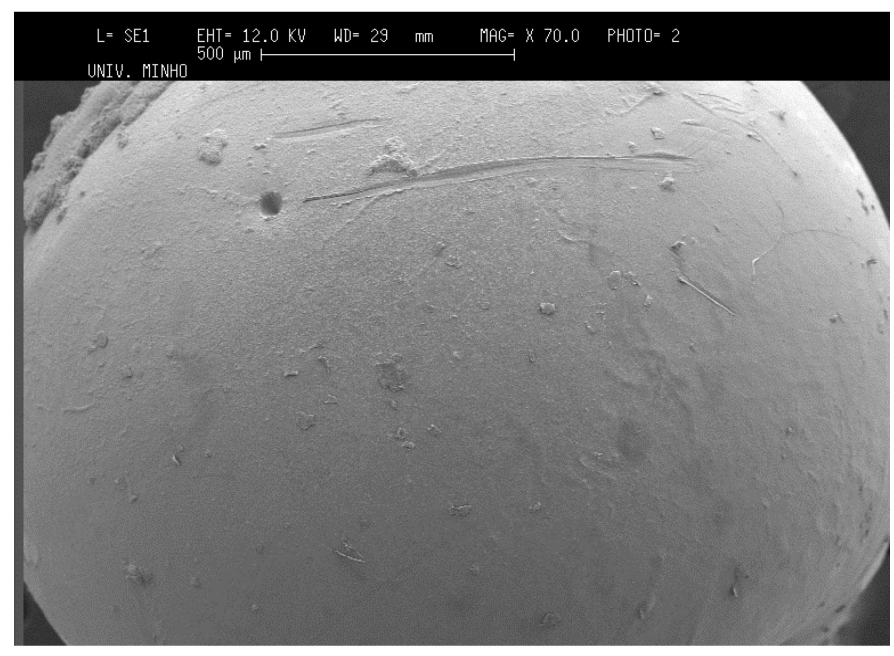

(b)

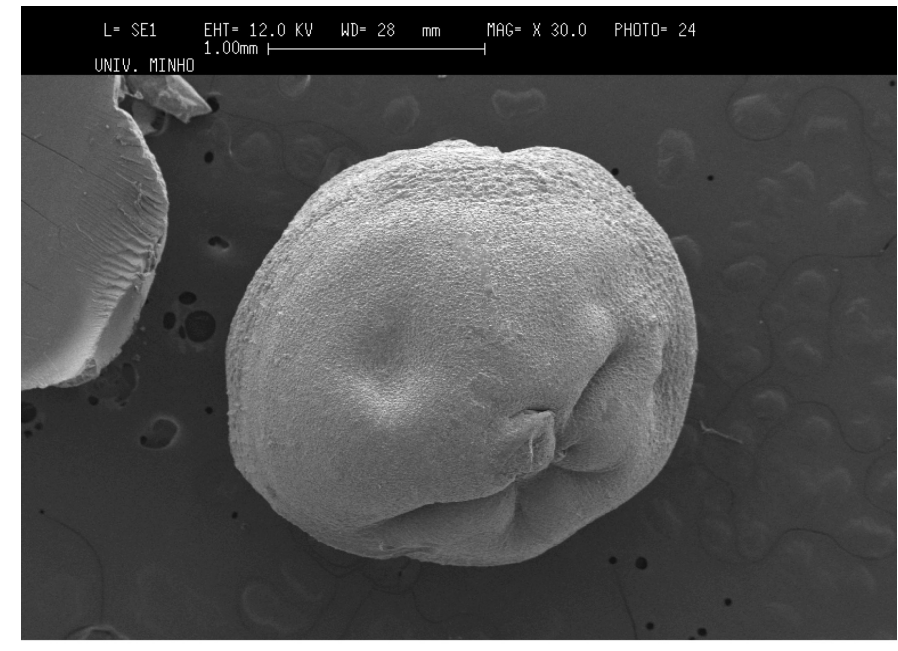

(c)

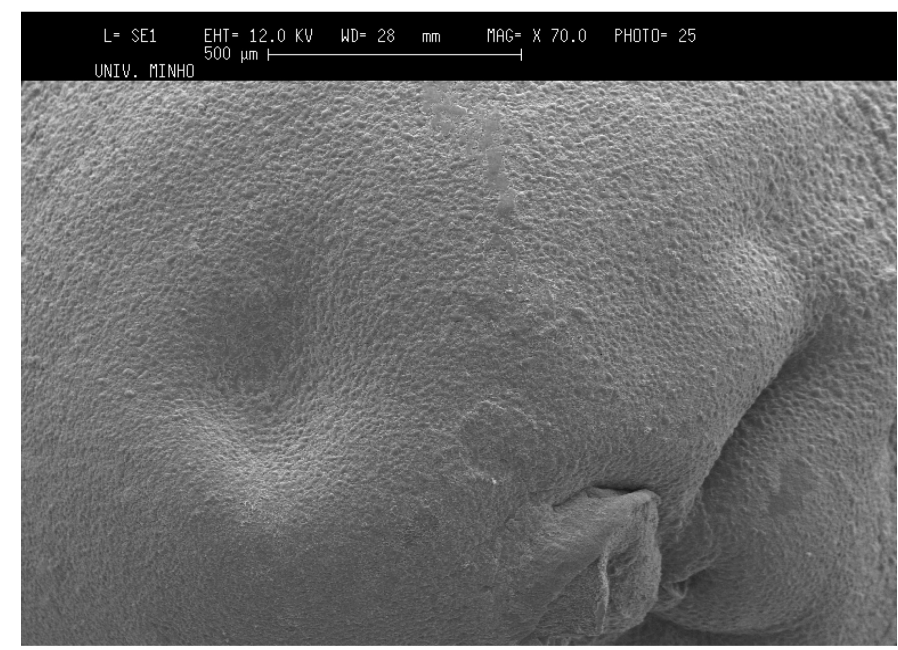

(d)

FIG. 2. SEM pictures of (a) and (b) CS beads without drug and (c) and (d) TCS beads without drug in different magnifications.

$\left(-\mathrm{CH}_{2}-\mathrm{OH}\right)$. The adsorption peaks at $3434 \mathrm{~cm}^{-1}$ is due to $-\mathrm{NH}$ stretching. The peaks at 1646 and $1578 \mathrm{~cm}^{-1}$ are due to the formation of amide linkage (-NHCO linkage). The peaks at 1254,1154 , and $896 \mathrm{~cm}^{-1}$ are due to thiol groups. The above peaks confirmed the structure of TCS.

\section{SEM of Prepared TCS Beads}

Figure 2 shows SEM images of drug-free CS and TCS beads. The liquid-gel transition of TCS in TPP aqueous solution can be explained by the electrostatic interaction between positively charged free amino groups with negatively charged counteranion TPP (Mi et al. 1999). The CS beads have a regular spherical shape, smooth surface, and homogeneous polymer matrix. This may be because cross-linking is more efficient in the CS beads.
The TCS beads have some cracks on the surface that may be due to the grafting of thiol-containing monomer. The insufficient cross-linking of TCS beads as the amino groups of CS were substituted by thiol compound has lead to the formation of rough and nonuniform surface structure. The size of the CS and TCS beads was found to vary in between $1.5-1.75 \mathrm{~mm}$.

\section{Swelling Behaviors of Beads}

The swelling behavior of the CS and TCS beads in solutions of $\mathrm{pH} 1.4$ and 7.4 at $37^{\circ} \mathrm{C}$ are shown in Figures 3 and 4. The TCS beads at $\mathrm{pH} 1.4$ were found to disintegrate within 3 days and then dissolve. The CS beads slowly swell and were found to be dissolved after 4 days. The results could be associated with differences in cross-linking density (Lee et al. 2001). 


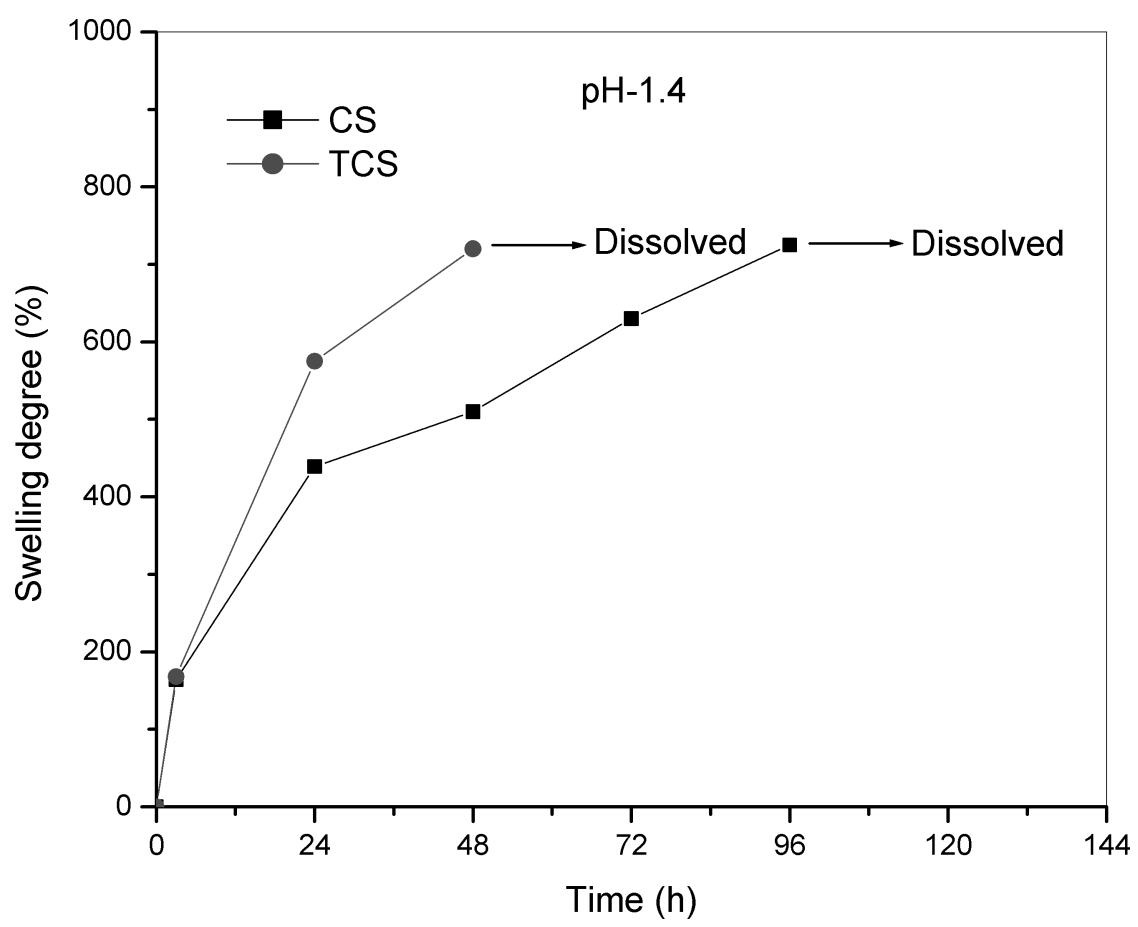

FIG. 3. Swelling behavior of CS and TCS beads at $\mathrm{pH} 1.4$.

The lower cross-linked TCS beads swelled quickly and gradually dissolved within 3 days, whereas the higher cross-linked beads did not dissolve within the same time. This is expected behavior, attributed to the high amount of interchain linkages in the well cross-linked chitosan-TPP network. The swelling per- centage was much higher at $\mathrm{pH} 1.4$ than $\mathrm{pH}$ 7.4. Moreover, the swelling reached a stable equilibrium much more rapidly in $\mathrm{pH}$ 7.4 than in $\mathrm{pH}$ 1.4. This is due to the lack of protonation of amino groups at $\mathrm{pH}$ 7.4, which prevents swelling in alkaline medium (Gupta and Ravi Kumar 2000).

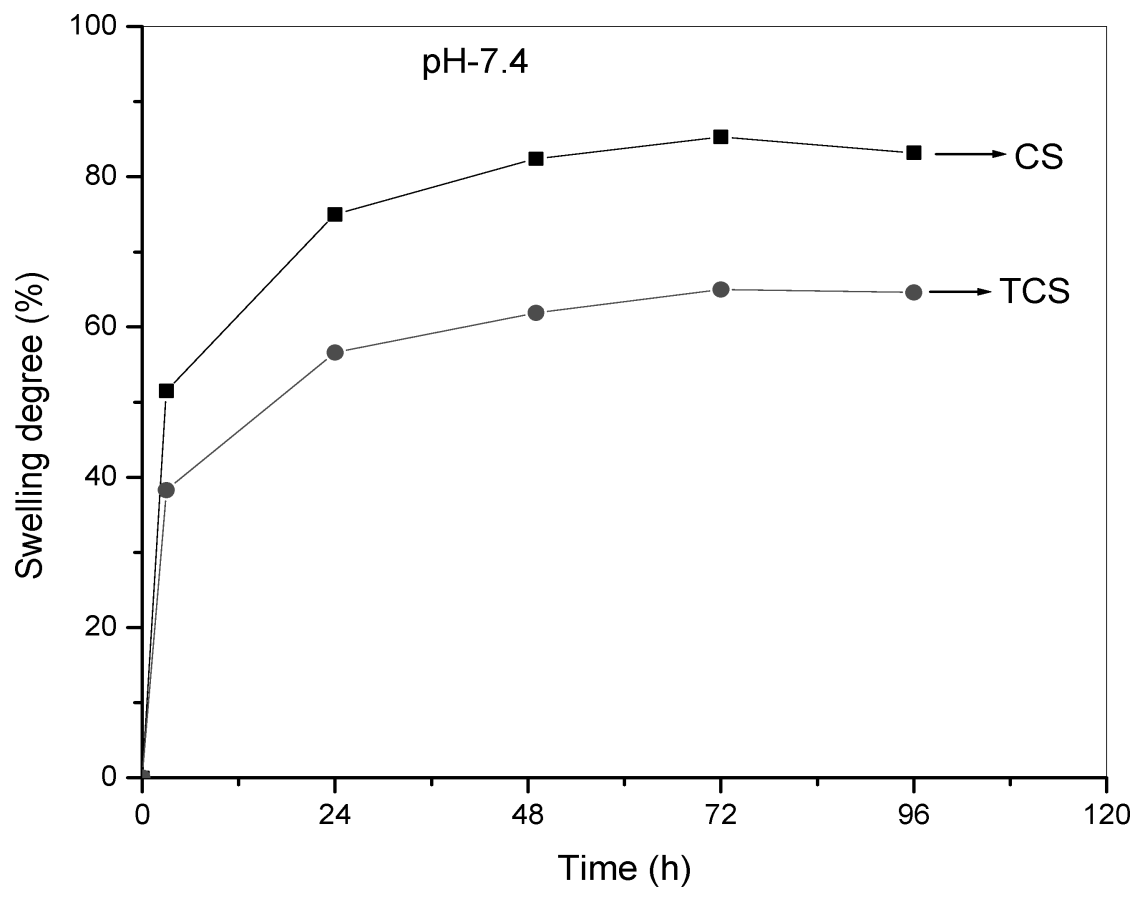

FIG. 4. Swelling behavior of CS and TCS beads at $\mathrm{pH}$ 7.4. 
TABLE 1

Loading efficiencies for IM-loaded CS and TCS Beads

\begin{tabular}{lccc}
\hline Formulation & $\begin{array}{c}\text { Initial drug } \\
\text { content } \\
\text { (mg/g-beads) }\end{array}$ & $\begin{array}{c}\text { Loading } \\
\text { level } \\
\text { (mg/g-beads) }\end{array}$ & $\begin{array}{c}\text { Loading } \\
\text { efficiency } \\
(\%)\end{array}$ \\
\hline $0.3 \%$ IM-CS & 28.9 & 27.1 & 93.81 \\
$0.6 \%$ IM-CS & 58.2 & 53.2 & 91.43 \\
$0.3 \%$ IM-TCS & 29.7 & 26.2 & 88.28 \\
$0.6 \%$ IM-TCS & 59.4 & 50.7 & 85.47 \\
\hline
\end{tabular}

$\mathrm{Im}=$ indomethacin, $\mathrm{CS}=$ chitosan, $\mathrm{TCS}=$ thiol-containia/chitosau.

\section{Entrapment of Drug in TCS Beads}

IM drug was efficiently entrapped into TCS beads during ionotropic process (see Table 1). The relatively high efficiency of loading could be explained by the low water solubility of IM as well as the ionic binding between the carboxyl group of IM and the thiol group in TCS beads. The carboxyl groups in IM and the thiol group in TCS are ionized at $\mathrm{pH} 4$. In this work, the drug was not chemically attached to the polymer. Thus, there will be only both electrostatic and hydrophobic interactions as well as the effect within the polymer matrix. Therefore, the drug is expected in a biologically active form and can exert its therapeutically effect upon the body as soon as it is released from the polymeric matrix.

\section{Im In Vitro Release Profiles}

Figure 5 shows the release profiles of $0.3 \%$ of IM-TCS beads at various $\mathrm{pH}$, at $37^{\circ} \mathrm{C}$. Within $3 \mathrm{hr}, 27 \%$ of IM was released from TCS beads at $\mathrm{pH} 1.4$ and $44 \%$ at $\mathrm{pH}$ 7.4. These results showed that the drug release profiles of TCS are in fact $\mathrm{pH}$ sensitive. Moreover, the more noticeable changes occur within a narrow $\mathrm{pH}$ range between 5.8 and 7.4. The following factors are to be considered in the noticeably higher release rate of IM at $\mathrm{pH} 7.4$ than that at $\mathrm{pH} 1.4$. The first factor is the higher solubility of IM in alkaline medium. The second factor is an electrostatic and hydrophobic repulsion between the carboxyl group of IM and the methyl group of modified TCS in pH 7.4 media (Hadgraft and Valenta 2000; Mi et al. 2002). This study shows that the percentages of drug release increase with an increasing $\mathrm{pH}$ in dissolution medium. The similar drug release profile also was observed for the CS beads. However, the release percentage of IM at $\mathrm{pH} 1.4$ was found to be less than that of TCS beads. The reason may be the formation of complexes between carboxyl group of IM and amino group of CS and poor solubility of drug at $\mathrm{pH}$ 1.4 (Mi et al. 2002). The velocity of diffusion of drug molecules is higher in the alkaline medium, effectively contributing to the controlled drug release. The polymer matrix probably combines two types of mechanisms for drug release: controlled diffusion and swelling.

Thiolated chitosans also display, besides their strong mucoadhesive and permeation-enhancing properties, excellent cohesive properties. The reducing thiol functions on the chitosan backbone enable the glycol proteins to also form inter- as well

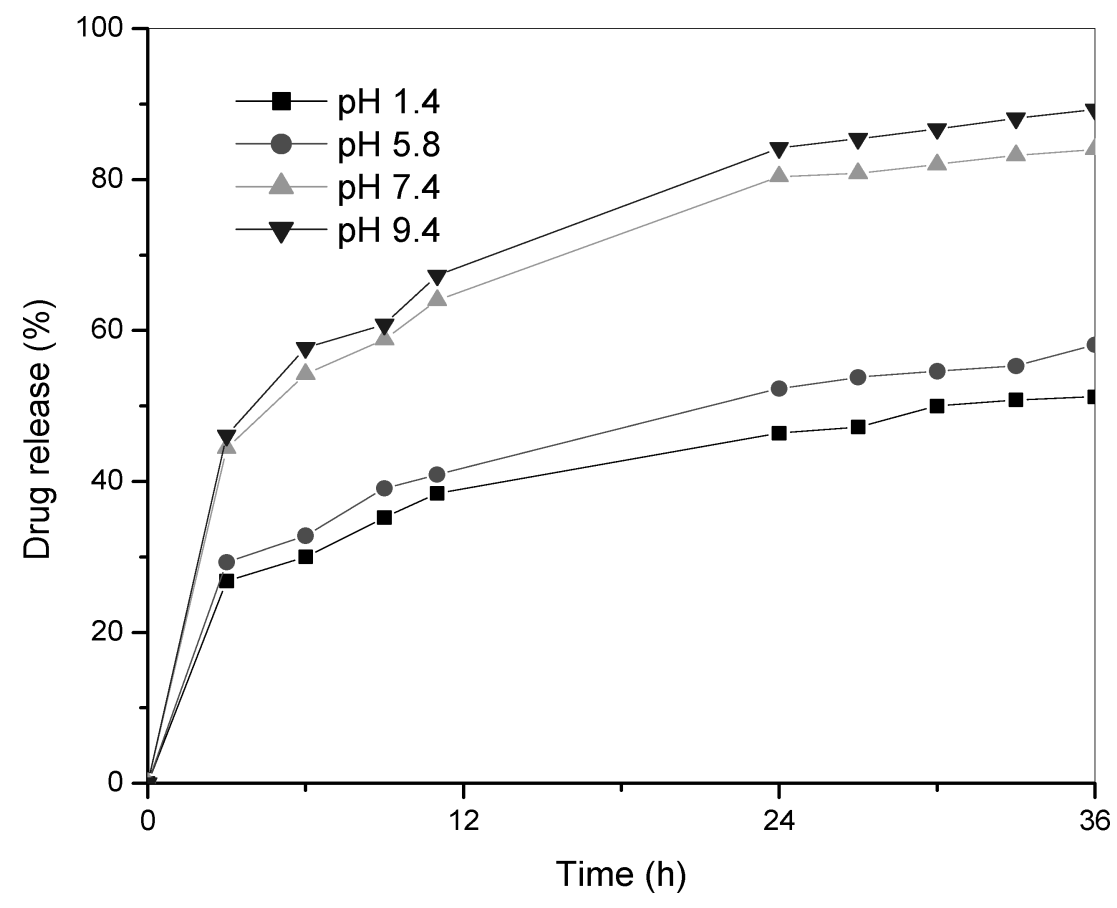

FIG. 5. Release profiles of IM from $0.3 \%$ loaded TCS beads in various $\mathrm{pH}$ media. 


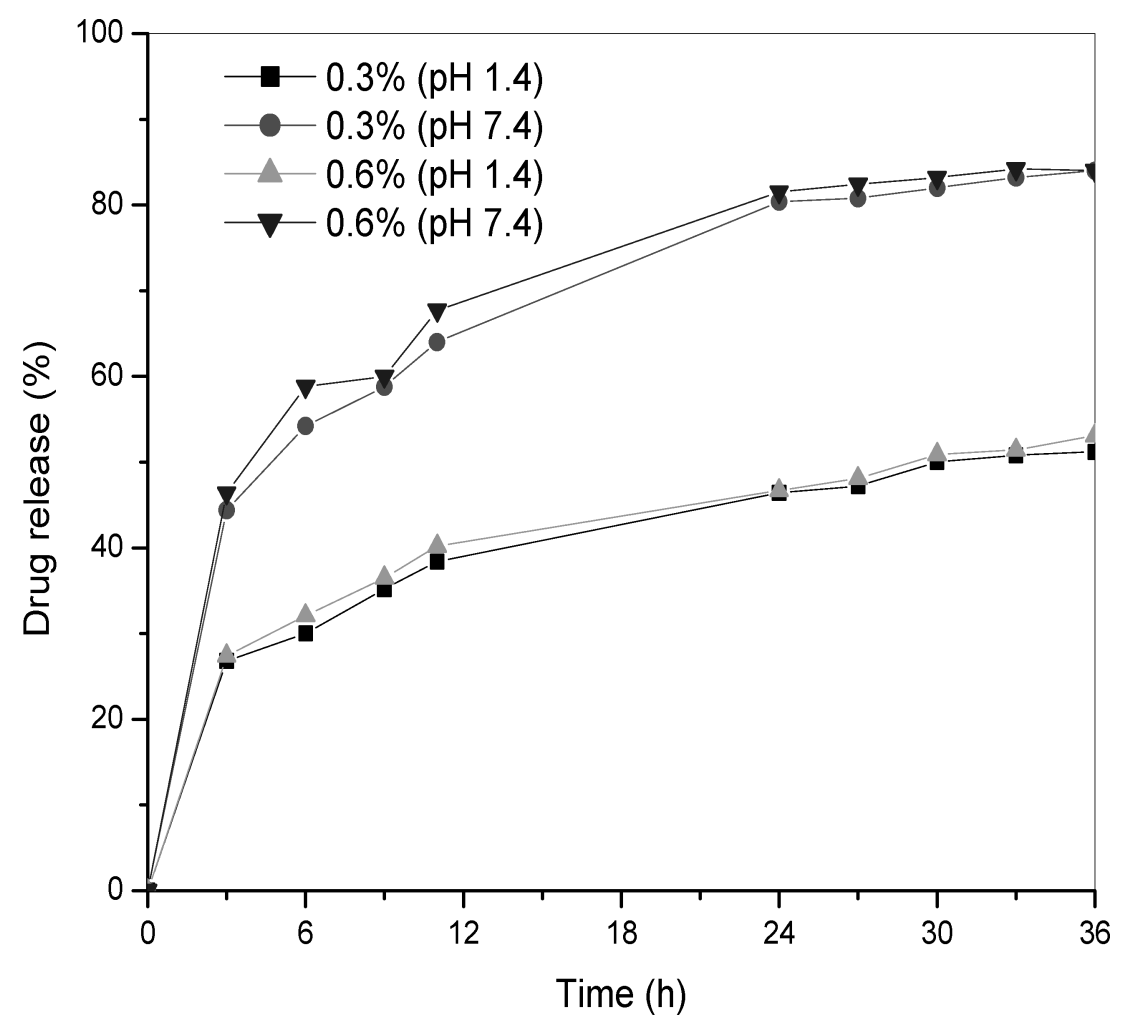

FIG. 6. Drug release from 0.3 and $0.6 \%$ of IM-loaded TCS beads in simulated gastric fluid, $\mathrm{pH} 1.4$, and simulated intestinal fluid, $\mathrm{pH}$ 7.4.

as intramolecular disulfide bonds. Such a cross-linking of the polymeric chains results in a high stability of drug carrier systems based on thiolated chitosans (Schnurch, Hornof, and Guggi 2004). Figure 6 shows the influence of the initial drug loading on the release percent of IM drug from TCS beads at $\mathrm{pH} 1.4$ and 7.4. There is no significant change between the release trends of 0.3 and $0.6 \%$ IM-loaded TCS beads. The amount of drug released from high drug-loaded beads is higher than that from low

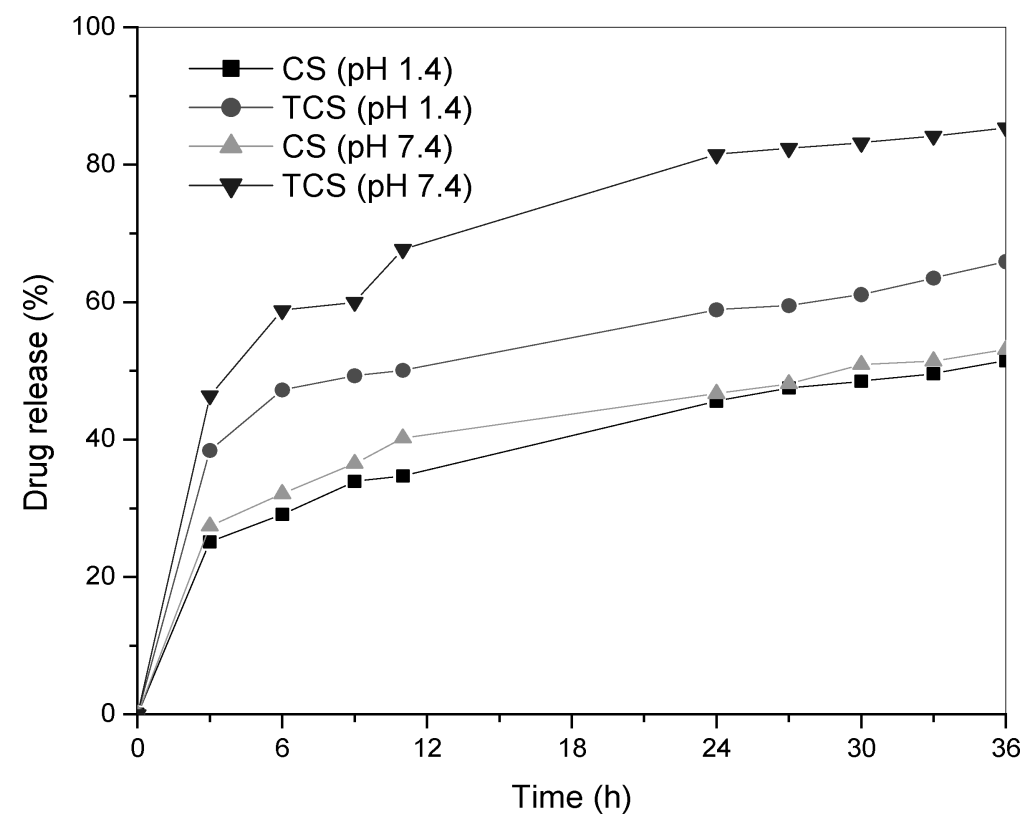

FIG. 7. Release profiles for initially $0.6 \%$ IM-loaded CS and TCS beads in simulated gastric fluid, $\mathrm{pH} 1.4$, and simulated intestinal fluid, $\mathrm{pH} 7.4$. 
drug-loaded beads. Increasing the initial drug content provides the larger equilibrium amount of drug released in each $\mathrm{pH}$.

Figure 7 shows the in vitro release profiles of $0.6 \%$ IMloaded CS and TCS beads investigated at $\mathrm{pH} 1.4$ and 7.4. At $\mathrm{pH} \mathrm{1.4,} \mathrm{25 \%} \mathrm{of} \mathrm{IM} \mathrm{was} \mathrm{released} \mathrm{from} \mathrm{CS} \mathrm{beads} \mathrm{within} 3 \mathrm{hr}$ while for TCS beads the amount of IM released was higher (27\%). Fully $38 \%$ of IM was released from CS beads at $\mathrm{pH} 7.4$ within $3 \mathrm{hr}$ while this value is increased to $44 \%$ of IM for TCS beads. In the case of CS beads, the complex is formed between the amino group of CS and carboxyl group of IM. In the TCS beads there is a hydrophobic interaction between the carboxyl group of IM and the methyl group of thiol-containing chitosan (Mi et al. 2002). TCS beads released the IM drug much faster than that of CS beads. When thinking of drug delivery applications, these improved mucoadhesive properties along with a controlled release out of the polymer matrix should render TCS beads very promising tools. Their positive result is due to the expected increase of drug bioavailability by adhering to mucosal tissues. Because of these properties, TCS beads should be further investigated.

\section{CONCLUSION}

Novel TCS beads have been synthesized for use as a controlled drug delivery system. The beads were prepared under mild conditions at room temperature at $\mathrm{pH} 4.0$, and the loading efficiency of a model drug, indomethacin, was over $85 \%$. The TCS beads were found to be $\mathrm{pH}$ sensitive. The water solubility of the drug, the swelling degree of beads, and the ionization of thiol groups influenced the release profiles of the drug at various $\mathrm{pHs}$. The release rate in simulated intestinal fluid $(\mathrm{pH}$ 7.4) is higher than that in simulated gastric fluid ( $\mathrm{pH} 1.4)$, enabling the drug delivery or release to take place preferentially in the intestine, avoiding drug leakage in the stomach. According to our results, TCS beads represent a promising tool as successful drug carriers for controlled drug delivery systems.

\section{REFERENCES}

Borchard, G. 2001. Chitosans for gene delivery. Adv. Drug Del. Rev. 52:145-150. Chen, S., and Wang, Y. 2001. Study on $\beta$-cyclodextrin grafting with chitosan and slow release of its inclusion complex with radioactive iodine. J. Appl. Polymer Sci. 82:2414-2421.

Clausen, A. E., Kast, C. E., and Schnurch, A. B. 2002. The role of glutathione in the permeation enhancing effect of thiolated polymers. Pharm. Res. 19:602608.

Felt, O., Furrer, P., Mayer, J. M., et al. 1999. Topical use of chitosan in ophthalmology: tolerance assessment and evaluation of precorneal retention. Int. J. Pharm. 180:185-193.

Fini, A., and Orienti, I. 2003. The role of chitosan in drug delivery: current and potential applications. Am. J. Drug Del. 1:43-59.

Gupta, K. C., and Ravi, Kumar, M. N. V. 2000. Drug release behavior of beads and microgranules of chitosan. Biomaterials 21:1115-1119.

Hadgraft, J., and Valenta, C. 2000. pH, pKa and dermal delivery. Int. J. Pharm. 200:243-247.

Heras, A., Rodriguez, N. M., and Ramos, V. M. 2001. $N$-methylene phosphonic chitosan: a novel soluble derivative. Carbohydrate Polymers 44:1-8.
Hoffman, A. S., Chen, G., Wu, X., et al. 1997. Graft copolymers of PEO-PPOPEO triblock polyethers on bioadhesive polymer backbones: synthesis and properties. Polymer Preprints 38:524-525.

Hornof, M., Kast, C. E., and Schnurch, A. B. 2003. In vitro evaluation of the viscoelastic behavior chitosan-thioglycolic acid conjugates. Eur. J. Pharm. Biopharm. 55:185-190.

Jayakumar, R., Prabaharan, M., Reis, R. L., and Mano, J. F. 2005. Graft copolymerized chitosan: present status and applications. Carbohydrate Polymers 62:142-158.

Jung, B. O., Kim, C. H., Choi, K. S., et al. 1999. Preparation of amphiphilic chitosan and their antimicrobial activities. J. Appl. Polymer Sci. 72:17131719.

Kast, C. E., and Schnurch, A. B. 2001. Thiolated polymers-thiomers: development and in vitro evaluation of chitosan-thioglycolic acid conjugates. Biomaterials 22:2345-2352.

Kast, C. E., Valenta, C., Leopold, M., and Schnurch, A. B. 2002. Design and in vitro evaluation of a novel bioadhesive vaginal drug delivery system for clotrimazole. J. Control. Rel. 81:347-354.

Kotze, A. F., Luessen, H. L., Leeuw, B. J. D., et al. 1997. $N$-Trimethyl chitosan chloride as a potential absorption enhancer across mucosal surfaces: in vitro evaluation in intestinal epithelial cells (CaCo-2). Pharm. Res. 14:11971202.

Kulkarni, A. R., Soppimath, K. S., and Aminabhavi, T. M. 1999. Controlled release of diclofenac sodium from sodium alginate beads crosslinked with glutaraldehyde. Pharma. Acta Helv. 74:29-36.

Kulkarni, A. R., Soppimath, K. S., and Aminabhavi, T. M. 2001. In-vitro release kinetics of cefadroxil-loaded sodium alginate interpenetrating network beads. Eur. J. Pharm. Biopharma. 51:127-133.

Lee, S. T., Mi, F. L., Shen, Y. J., Shyu, S. S. 2001. Equilibrium and kinetic studies of copper(II) ion uptake by chitosan-tripolyphosphate chelating resin. Polymer 42:1879-1892.

Liu, G. W., and Yao, K. D. 2002. Chitosan and its derivatives-a promising non-viral vector for gene transfection. J. Control. Rel. 83:1-11.

Mi, F. L., Shyu, S. S., Chen, C. T., and Lai, J. Y. 2002a. Adsorption of indomethacin onto chemically modified chitosan beads. Polymer 43:757765.

Mi, F. L., Shyu, S. S., Lee, S. T., and Wong, T. B. 1999. Kinetic study of chitosan-tripolyphosphate complex reaction and acid-resistive properties of the chitosan-tripolyphosphate gel beads prepared by in-liquid curing method. J. Polymer Sci., Part-B. Polymer Physics 37:1551-1564.

Mi, F. L., Sung, H. W., and Shyu, S. S. 2002b. Drug release from chitosanalginate complex beads reinforced by a naturally occurring cross-linking agent. Carbohydrate Polymers 48:61-72.

Muzzarelli, R. A. A. 1997. Cell Molecular and Life Science. 53:131-140.

Ono, K., Saito, Y., Yura, H., and Ishikawa, K. 2000. Photocrosslinkable chitosan as a biological adhesive. J. Biomed. Mat. Res. 49:289-295.

Sashiwa, H., and Shigemasa, Y. 1999. Chemical modification of chitin and chitosan 2: preparation and water soluble property of $\mathrm{N}$-acylated or $\mathrm{N}$ alkylated partially deacetylated chitins. Carbohydrate Polymers 39:127138.

Schnurch, A. B., Brandt, U. M., and Clausen, A. E. 1999. Synthesis and in vitro evaluation of chitosan-cysteine conjugates. Sci. Pharm. 67:196-208.

Schnurch, A. B., and Hornof, T. E. 2001. Synthesis and in vitro evaluation of chitosan-thioglycollic acid conjugates. Sci. Pharm. 69:109-118.

Schnurch, A. B., Hornof, M., and Guggi, D. 2004. Thiolated chitosans. Eur. J. Pharm. Biopharm. 57:9-17.

Schnurch, A. B., Hornof, M., and Zoidl, M. 2003. Thiolated polymersthiomers:modification of chitosan with 2-iminothiolane. Int. J. Pharm. 260:229-237.

Senel, S., Kremer, M., Kas, S., et al. 2000. Enhancing effect of chitosan on peptide drug delivery across buccal mucosa. Biomaterials 21:20672071.

Singh, D. K., and Ray, A. R. 1998. Characterization of grafted chitosan films. Carbohydrate Polymers 36:251-255. 
Sorlier, P., Denuziere, A., Viton, C., and Domard, A. 2001. Relation between the degree of acetylation and the electrostatic properties of chitin and chitosan. Biomacromolecules 2:765-772.

Sugimoto, M., Morimoto, M., and Sashiwa, H. 1998. Preparation and characterization of water-soluble chitin and chitosan derivatives. Carbohydrate Polymers 36:49-59.

Takeuchi, H., Yamamoto, H., and Kawashima, Y. 2001. Mucoadhesive nanoparticulate systems for peptide drug delivery. Adv. Drug Del. Rev. 47:39-54.

Tasker, R. A., Connel, B. J., Ross, S. J., and Elson, C. M. 1998. Development of an injectable sustained-release formulation of morphine: antinociceptive properties in rats. Lab. Animals 32:270-275.
Thanou, M., Verhoef, J. C., and Junginger, H. E. 2001. Oral drug absorption enhancement by chitosan and its derivatives. Adv. Drug Del. Rev. 52:117126.

Xie, W. M., Xu, P. X., Wang, W., Liu, Q. 2001. Antioxidant activity of watersoluble chitosan derivatives. Biomat. Medi. Chemi. Lett. 13:1699-1701.

Xie, W. M., Xu, P. X., Wang, W., and Liu, Q. 2002. Preparation and antibacterial activity of a water-soluble chitosan derivative. Carbohydrate Polymers 50:3540.

Yang, Z. K., and Yuan, Y. 2001. Studies on the synthesis and properties of hydroxyl azacrown ether-grafted chitosan. J. Appl. Polymer Sci. 82:18381843. 